\title{
Modeling of the Function Principle of Semiconductor Gas Sensors for High School Students
}

\author{
https://doi.org/10.3991/ijoe.v17i03.19213 \\ Sebastian Höfner ${ }^{\bowtie}$, Andreas Schütze \\ Saarland University, Saarbrücken, Germany \\ s.hoefnerelmt.uni-saarland.de \\ Michael Hirth, Jochen Kuhn \\ Technical University Kaiserslautern, Kaiserslautern, Germany \\ Benjamin Brück \\ Student Research Center, Saarlouis, Germany
}

\begin{abstract}
A wide range of pollutants cannot be perceived with human senses, which is why the use of gas sensors is indispensable for an objective assessment of air quality. Since many pollutants are both odorless and colorless, there is a lack of awareness, in particular among high school students. The project SUSmobil (funded by DBU - Deutsche Bundesstiftung Umwelt) aims to change this. In three modules on the topic of gas sensors and air quality, the students are familiarized with the functionality of a metal oxide semiconductor (MOS) gas sensor, perform a sensor calibration and carry out environmental measurements with calibrated sensors. Based on these introductory experiments, the students are encouraged to develop their own environmental questions. In this contribution, we focus on the experimental and modeling approach which explains the function principle of a MOS gas sensor in a way suitable for high school students. This includes a qualitative and quantitative description of a simplified sensor model explaining the main processes on the sensor surface. In addition, an HTML-based self-learning course is presented in which the students investigate the sensor behavior in the presence of different substances depending on the sensor temperature.
\end{abstract}

Keywords-Air pollution; metal oxide semiconductor gas sensors; student experiment; education; volatile organic compounds; high school

\section{Introduction and Motivation}

Air pollution is the single largest environmental health risk in Europe with over 400.000 deaths per year in 2018 [1]. According to the World Health Organization (WHO) air pollution is a major cause for heart diseases and strokes, as well as lung diseases and even Alzheimer's [2]. Inside buildings indoor air pollutants are responsible for the sick building syndrome with symptoms like headache and nausea [3]. 
The awareness of air pollutants has increased in recent years, as can be seen by the "Fridays For Future" movement, but especially young people often have a diffuse or only vague idea of it. For example, there is a widespread misconception about carbon dioxide $\left(\mathrm{CO}_{2}\right)$ and its role as pollutant [4]. Although it is harmful to the environment as a greenhouse gas and contributes significantly to climate change in addition to gases like methane $\left(\mathrm{CH}_{4}\right)$, for human beings it is only dangerous at very high concentrations (>1\% continuous exposure over more than three weeks), with symptoms like increased respiratory rate, dizziness, confusion and dyspnea [5]. Typically, these high concentrations are never reached indoors. Nevertheless, $\mathrm{CO}_{2}$ can serve as indicator for poor indoor air quality, because other pollutants like volatile organic compounds (VOC) correlate with the $\mathrm{CO}_{2}$ concentration, when there is no other source of VOCs besides humans [6]. This has led to the widely accepted Pettenkofer value of 1000 ppm for $\mathrm{CO}_{2}$, above which increased ventilation is recommended [7].

To increase awareness about air quality, the outreach project "SUSmobil" (German: Schüler-Umwelt-Studien mit mobilen Messgeräten, English: Environmental Studies by Students with mobile measuring devices) aims to teach students, age 12 to 18, about air quality and how it is determined with low-cost sensors [8]. In three modules the students learn about the function principle of metal oxide semiconductor (MOS) gas sensors (module 1), the required calibration process for quantification of target gas concentrations (module 2) and finally perform practical measurements of indoor air quality (IAQ, module 3) [9]. These modules form the theoretical basis for students to develop their own environmental studies in the form of citizen science projects. The range of participation possibilities for students within citizen science projects is diverse and ranges from the provision of resources and sensor data to the development of their own questions, methods and analytics as part of pure citizen science. [10]. Active participation in the research process can help demystify science as such and make it possible to experience the process of gaining scientific knowledge [11]. Examples of student environmental studies are investigations of particulate matter (PM) emissions near a school, of the influence of plants on bedroom air quality or of the air composition in beehives and the bees' reaction to increased $\mathrm{CO}_{2}$ levels [12].

The current paper focuses on module 1 - the function principle of a MOS gas sensor. First the physical sensor model, based on grain-grain-boundaries is described (section II). After that, a simplified student model is presented, which describes the sensor reaction as a superposition of three effects (section III). Then its performance and limitations compared to the physical sensor model are discussed (section IV). Then, the experimental implementation for deriving the simplified sensor model is described which is based on an HTML-based self-learning course by investigating the sensor response in the presence of reducing gases (section V). In particular, the temperature dependence of the sensor reaction is addressed. Section VI concludes this contribution with a brief summary and outlook. 


\section{Physical Model of The Function Principle of Metal Oxide Semiconductor Gas Sensors}

Most metal oxide semiconductor (MOS) gas sensors are based on nsemiconducting tin dioxide $\left(\mathrm{SnO}_{2}\right)$, which is applied as a granular layer on the surface of a substrate. The description of the conduction mechanism is mainly reduced to the observation of grain-grain boundaries forming energy barriers for electrons in the conduction band [13], cf. Fig. 1. Depending on the sensor temperature, two different reactions based on the adsorption of oxygen on the surface can take place. According to [14] the important surface reaction for temperatures below approx. $150^{\circ} \mathrm{C}$ is

$$
\mathrm{O}_{2, a d s}+e^{-} \rightarrow \mathrm{O}_{2, a d s}^{-}
$$

while for temperatures above approx. $150^{\circ} \mathrm{C}$ oxygen dissociates

$$
\frac{1}{2} O_{2, a d s}+e^{-} \rightarrow O_{a d s}^{-}
$$

Thus, oxygen adsorption removes free charge carriers from the semiconductor, especially on the sensor surface.

Reducing gases, such as carbon monoxide $(\mathrm{CO})$, react with the adsorbed oxygen, thereby releasing charge carriers again.

$$
\mathrm{CO}+\mathrm{O}_{a d s}^{-} \rightarrow \mathrm{CO}_{2}+e^{-}
$$

The electron-depleted zone near the surface decreases, reducing the energy barrier and increasing conductivity, which can be measured as the sensor reaction (3). In the band model, the depletion zone is described by the formation of an energy barrier $E_{\mathrm{B}}$ (Schottky barrier) at the grain-grain boundaries, see Fig. 1.

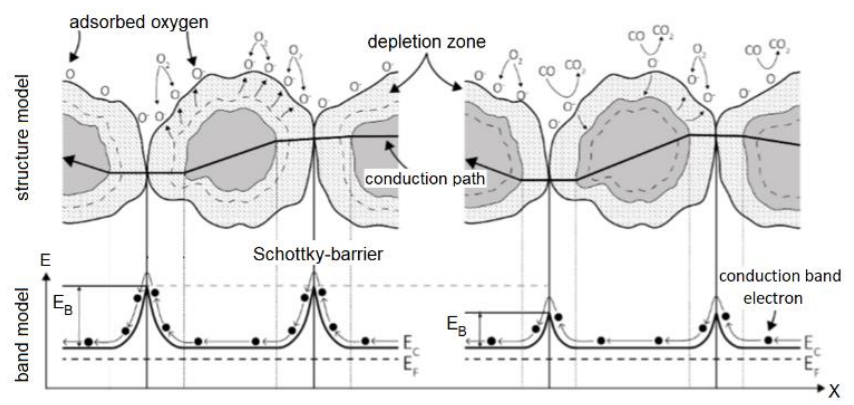

Fig. 1. Model for describing the functionality of a metal oxide semiconductor gas sensor based on the grain-grain boundaries. Schematic representation as structure (top) and band model (bottom) of a polycrystalline layer of a MOS gas sensor in air (left) and in the presence of a reducing gas, here $\mathrm{CO}$ (right). $E_{\mathrm{C}}$ denotes the lowest energy of the conduction band, $E_{\mathrm{B}}$ indicates the Schottky barrier and $E_{\mathrm{F}}$ refers to the Fermi energy [13], [15]. 
According to [13] the description of the conductivity $\sigma$ of the sensor based on the grain boundary model can be reduced to the temperature-dependent function (4)

$$
\sigma=\sigma_{0} \cdot \exp \left(-\frac{E_{\mathrm{B}}}{k_{\mathrm{B}} T}\right)
$$

Here, $k_{\mathrm{B}}$ is the Boltzmann constant and $T$ the temperature of the semiconducting material. The energy barrier $E_{\mathrm{B}}$ depends on the surface charge density $N_{\mathrm{S}}$ of adsorbate states of oxygen and the donor density $N_{\mathrm{D}}$. By using the one-dimensional Poisson equation with Schottky approximation and the Schottky potential $V_{\mathrm{S}}$, a relation for the energy barrier (Schottky barrier) can be found [13]

$$
E_{\mathrm{B}}=e V_{\mathrm{S}}=\frac{e^{2} N_{\mathrm{S}}^{2}}{2 \varepsilon_{\mathrm{r}} \varepsilon_{0} N_{\mathrm{d}}}
$$

including the elementary charge $e$, and the permittivities of vacuum $\varepsilon_{0}$ and of tin dioxide $\varepsilon_{\mathrm{r}}$ respectively. In dynamic equilibrium the surface charge density $N_{S}$ of adsorbate states with an energy $E_{\mathrm{A}}$ depends on the Fermi energy $E_{\mathrm{F}}$ and the temperature. According to [14] it can be described in equilibrium by the Fermi distribution

$$
N_{S} \sim \frac{1}{1+\exp \left(\frac{E_{A}-E_{F}}{k_{B} T}\right)}
$$

The pre-factor $\sigma_{0}$ of conductivity (4) is proportional to the electron mobility $\mu_{\mathrm{S}}$ and the donor density $N_{\mathrm{d}}$ [14]. It is linked to the electrical conductance $G_{0}$ via a geometric factor $A_{\text {geom }}$

$$
\frac{G_{0}}{A_{\text {geom }}}=\sigma_{0}=e \cdot \mu_{S} \cdot N_{d}
$$

The donor density $N_{\mathrm{d}}$ in $\mathrm{SnO}_{2}$ is caused by single and double ionized oxygen defects in the bulk. The energies for the first and second ionization are approx. $30 \mathrm{meV}$ and $150 \mathrm{meV}$, respectively [15]. At typical sensor operating temperatures $\left(200-440{ }^{\circ} \mathrm{C}\right.$ ), corresponding to average thermal energies between 40 and $60 \mathrm{meV}$, the donor states of the first ionization are almost completely depleted, while the states of the second ionization are still almost completely occupied. Thus, $N_{\mathrm{d}}=$ const. is a valid assumption in the operating range of the sensor [14]. Due to the large band gap of $\mathrm{SnO}_{2}$ of approx. $3.7 \mathrm{eV}$, no intrinsic conductivity is to be expected, hence the conductivity is exclusively due to ionized donors [16].

Due to collisions of the electrons with lattice defects and phonons (quantized lattice oscillations), the electron mobility decreases at higher temperatures according to the relationship [13]

$$
\mu_{\mathrm{S}} \sim T^{-3 / 2}
$$

This results in a temperature-dependent conductivity in the form

$$
\sigma \sim T^{-3 / 2} \cdot \exp \left(-\frac{E_{\mathrm{B}}}{k_{\mathrm{B}} T}\right)
$$


Using (5) and (6), overall, this leads to the expression

$$
\sigma \sim T^{-3 / 2} \cdot \exp \left(-\frac{1}{k_{\mathrm{B}} T\left(1+\exp \left(\frac{E_{A}-E_{F}}{k_{B} T}\right)\right)^{2}}\right)
$$

In case of a constant voltage, the electrical conductance $G$, conductivity $\sigma$ and the electrical current I are proportional to each other. Thus, $G$ and the electrical resistance $R=G^{-1}$ can be expressed qualitatively as a function of the sensor temperature by

$$
R=\frac{1}{G} \sim T^{3 / 2} \cdot \exp \left(\frac{1}{k_{\mathrm{B}} T \cdot\left(1+\exp \left(\frac{E_{\mathrm{A}}-E_{\mathrm{F}}}{k_{\mathrm{B}} T}\right)\right)^{2}}\right)
$$

Fig. 2 schematically shows the basic behavior of $G$ and $R$ as a function of the sensor temperature $T$ in pure air and in air with an additional reducing gas.
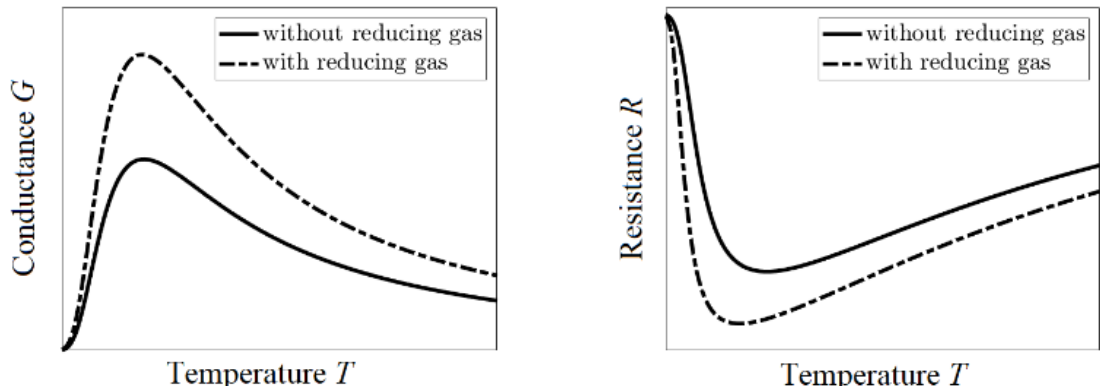

Fig. 2. Qualitative theoretical behavior of the electrical conductance $G$ (left) and resistance $R$ (right) of a MOS gas sensor in dynamic equilibrium as a function of the sensor temperature $T$ in pure air (dotted line) and in the presence of a reducing gas (solid line). The graphs are based on Eq. (11) with different bonding energies $E_{\mathrm{B}}$ according to Eq. (5).

\section{Simplified Model of the Function Principle - Qualitative and Quantitative Consideration}

Teaching the theoretical basics of the functionality of MOS gas sensors in high schools faces the challenge that concepts of semiconductor physics including the band model are not established and cannot be developed without great efforts. In addition, a high level of abstraction is inherent in the consideration of grain-grain boundaries and the dependence of conductivity on space-charge regions which are more or less depleted of electrons. Thus, a simplified model adapted to the level of learning and knowledge of high school students was developed. 
This model is intended to describe as adequately as possible

a) The sensor behavior at different operating temperatures

b) The processes taking place on the sensor surface with and without reducing gas as the core of the sensor reaction

This includes the explanation for the existence of a resistance minimum $R_{\min }$ (a) and of the decrease of the resistance in the presence of a reducing gas (b), cf. Fig. 2. In the following, the components of a mechanistic functional model are first described before model assumptions and implications with increasing complexity are presented qualitatively in three steps. The corresponding quantitative descriptions on which the presented graphs are based is included in the appendix.

\subsection{Model components, assumptions and predictions}

Fig. 3 shows the schematic structure of the developed sensor model. According to the simple Drude model [17], electrons, represented by grey dots, move within the sensor material as free charge carriers due to a constant electrical voltage $U_{0}$. Their movement in $x$-direction caused by the electric field is indicated by arrows, their length indicates their velocity $v=d x / d t$. Let $A$ be the cross-sectional area of the sensor.

By introducing the charge density $\rho=Q / V$, with the total charge $Q$ and the volume of the sensor material $V=A \cdot x$, the electrical current $I$ and conductance $\mathrm{G}$ can be derived according to (12).

$$
G \sim I=\frac{d Q}{d t}=\rho \cdot A \cdot \frac{d x}{d t}=\rho \cdot A \cdot v=\frac{Q}{x} \cdot v
$$

Assuming a homogeneous charge distribution $Q / x=$ const. the electric conductance $G$ is proportional to the product of the total charge $Q$ (or number of charge carriers) and the electron velocity $v$

$$
G \sim Q \cdot v
$$

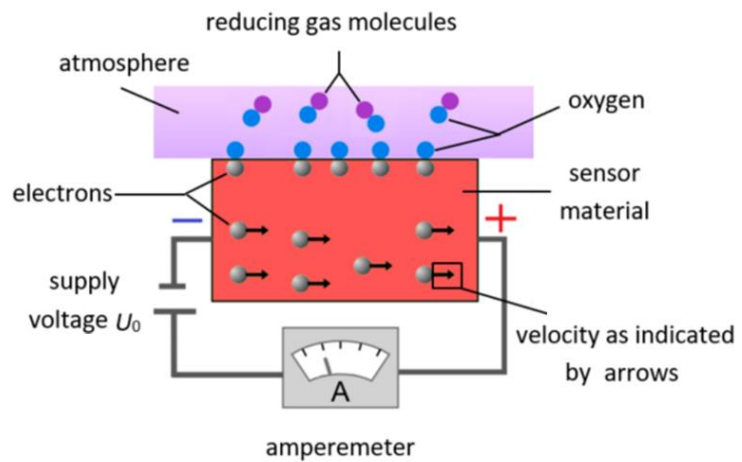

Fig. 3. Schematic structure and components of a mechanistic functional model for MOS gas sensors. 
Variable sensor temperatures are symbolized by the colors blue $=$ "cool $(\lesssim 100$ $\left.{ }^{\circ} \mathrm{C}\right)$ ", yellow $=$ "warm $\left(100{ }^{\circ} \mathrm{C}-250^{\circ} \mathrm{C}\right) "$ and red $=$ "hot $\left(\gtrsim 250{ }^{\circ} \mathrm{C}\right) "$. Above the sensor material the surrounding atmosphere is depicted, with oxygen and reducing gas molecules indicated by blue and violet dots, respectively. For simplification, no distinction is made between atomic $(\mathrm{O})$ and molecular $\left(\mathrm{O}_{2}\right)$ oxygen, and the gas molecules are also considered quasi atomic (indivisible).

\subsection{Effect of sensor temperature - No atmosphere considered}

The simplified model describes the effect of increasing temperature on the sensor as an increase in the effective electron velocity. At the same time, the total charge within the semiconductor without an external atmosphere remains constant. Due to the increase in electron velocity, the electrical conductance also increases with higher temperatures according to (13). Fig. 4 shows the influence of temperature in the simplified sensor model.
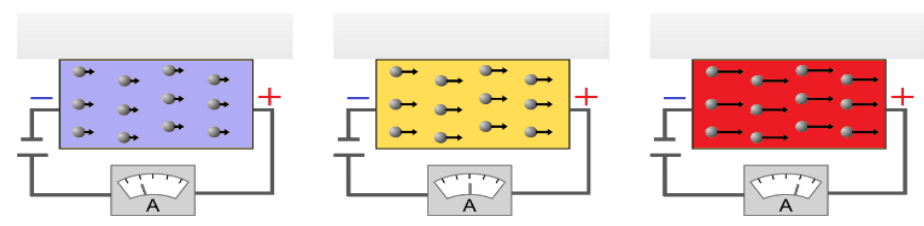

Fig. 4. Schematic representation of sensor behavior without external atmosphere at low (left), medium (center), and high (right) sensor temperatures. The velocity of the electrons in creases with temperature, while the number of charge carriers remains constant. Thus, the electrical conductance increases with increasing temperature.

The assumed dependence of the conductance on temperature corresponds to the exponential decrease of the resistance typical for an NTC thermistor, a widely used temperature sensor, cf. Fig. 5 [18]. This model presentation qualitatively replaces the temperature influence of the grain boundaries of the physical model. Similarly, the assumption of a fixed number of free electrons inside the sensor material resembles an electron density independent of temperature. 

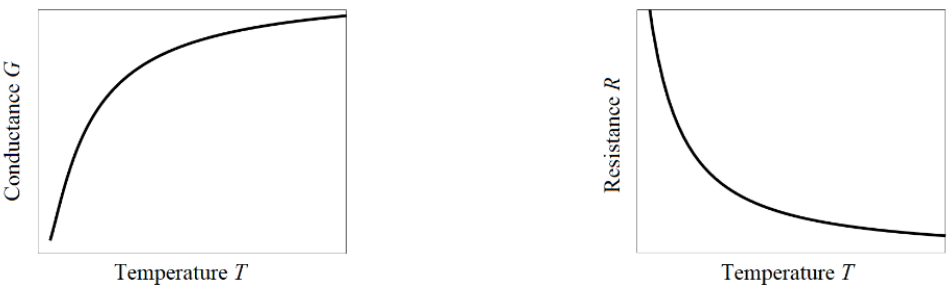

Fig. 5. Qualitative dependence of the electrical conductance (left) Qualitative dependence of the Resistance (right) of an NTC thermistor on temperature

\subsection{Influence of an oxygen atmosphere without reducing gas}

In the presence of an oxygen-containing atmosphere, the oxygen adsorbs on the sensor surface, capturing electrons, which are no longer available for charge transport. The higher the sensor temperature, the more adsorption processes take place, as these require an activation energy. This reduces the number of free electrons in the sensor material with increasing temperature. Combining the effects of electron velocity and oxygen adsorption, three cases are considered for various sensor temperatures, see Fig. 6.
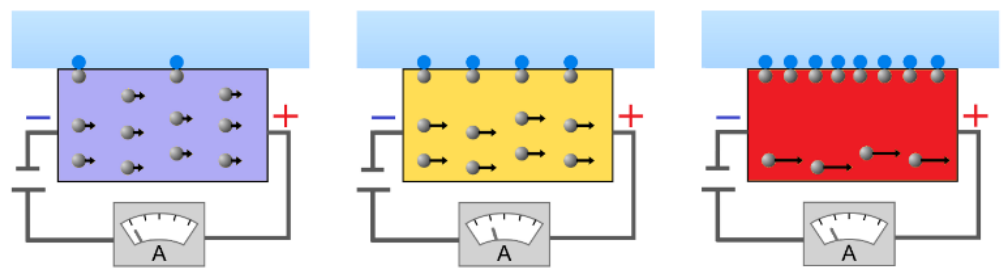

Fig. 6. Schematic representation of the sensor behavior in an atmosphere containing oxygen at low (left), medium (middle) and high (right) temperatures. The velocity of the electrons increases with temperatures, while the number of free electrons is reduced due to capturing by oxygen at the surface.

At low temperature, the low electron velocity leads to a low total current despite many electrons. At high temperature and high velocities only few free electrons are available, again resulting in a low total current. At medium temperature the influence of both competing effects results in a maximum total current. The electrical conductance shows the same behavior while the electrical resistance shows a minimum at medium temperature as depicted in Fig. 7. 


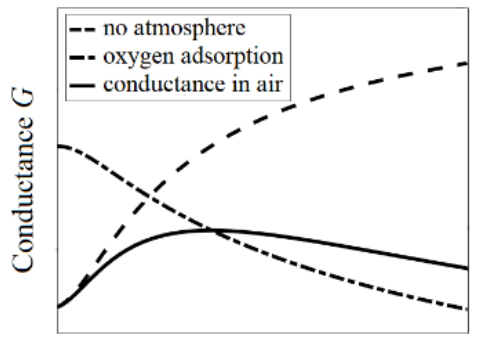

Temperature $T$

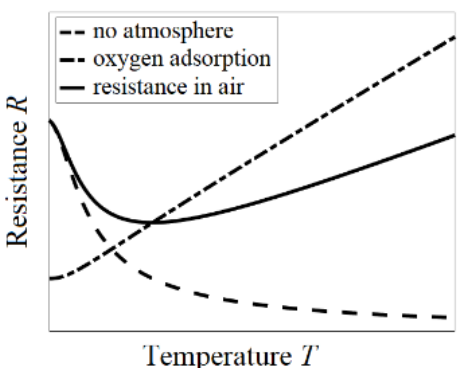

Temperature $T$

Fig. 7. Qualitative dependence of the electrical conductance (left) and resistance (right) of a MOS gas sensor as a result of the superposition of the two competing effects temperature and oxygen adsorption: the velocity of the electrons increases with temperature, while the number of free electrons is reduced due to adsorption of oxygen on the sensor surface.

\subsection{Influence of a reducing gas}

Reducing gas molecules in the atmosphere (e.g. CO or ethanol) can react with oxygen adsorbed on the surface, causing it to desorb. Electrons previously captured by the oxygen are released and are available again for charge transport. Thus, the electrical conductance increases, see Fig. 8.
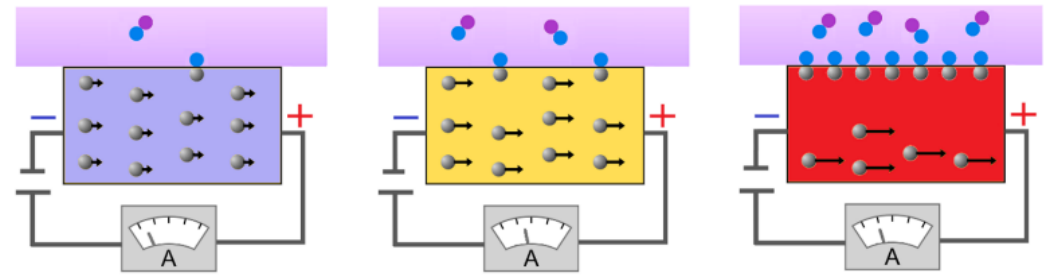

Fig. 8. Schematic representation of the sensor behavior in an atmosphere containing oxygen plus a reducing gas at low (left), medium (middle) and high (right) temperatures.

The reaction rate of the reducing gas with the oxygen depends on the concentration of the gas in the atmosphere, which is initially assumed to be constant, as well as on the oxygen coverage and the sensor temperature. The higher the temperature and the more oxygen is adsorbed, the greater the probability that the reducing gas will react with the bound oxygen. At the same time, the surface coverage with oxygen is limited due to the increasing number of negatively charged oxygen molecules (Weisz-Limit, [18]). Therefore, the surface coverage remains almost constant at very high temperatures, where the fast adsorption of oxygen dominates over the reaction with the reducing gas, decreasing the response to the reducing gas, see Fig. 9. 

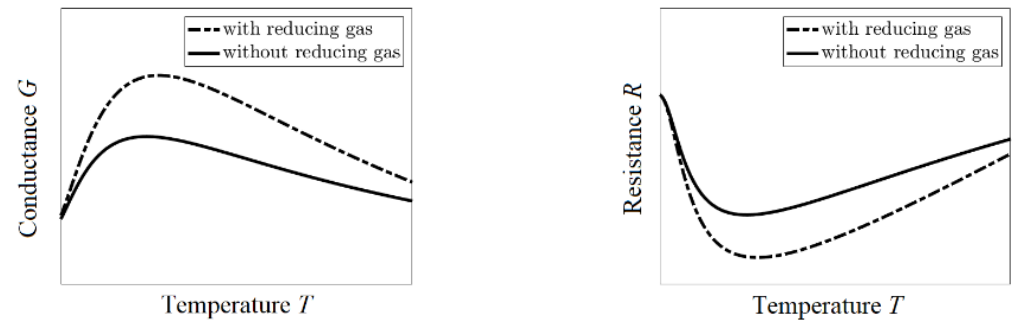

Fig. 9. The figures indicate that the response of the gas sensor to reducing gas is also temperature dependent. Influence of a reducing gas on the conductance $G$ (left), The electrical resistance $R$ (right) depending on the temperature

\section{$4 \quad$ Model Performance and Limits}

The simplified sensor model is able to describe the relevant factors for the functionality of a MOS gas sensor both qualitatively and semi-quantitatively on an education level suitable for high school students. This involves adsorption of oxygen on the sensor surface as well as the reaction with a reducing gas as essential components to understand the sensor reaction. The principle behavior of the electrical conductance or resistance as a function of the sensor temperature agree with the prediction of the physical theory based on the grain- boundary model as well as with the experimental observations (see section V). In particular, these include a maximum of the electrical conductance corresponding to a minimum of the electrical resistance at "medium" temperatures. Fig. 10 shows a comparison of the predictions of the electrical conductance and resistance vs. temperature according to the grain-boundary model and the simplified mechanistic sensor model. The curves of the simplified sensor model are based on the quantitative description as described in the appendix. Note that it is possible to extend the simplified sensor model also to oxidizing gases.
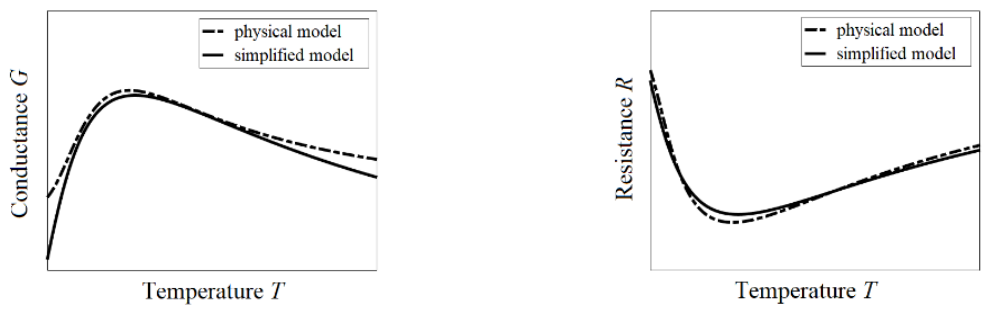

Fig. 10.Comparison of the qualitative predictions of the electrical conductance $G$ (left) and the electrical resistance R (right) according to the physical sensor model based on graingrain-boundaries (dotted line) and the simplified model (continuous line) based on (11) and (26). 
The simplified mechanistic model also adequately explains the shift of these curves in the presence of a reducing gas, as well as the effect that the values with and without reducing gas converge at very low and very high temperatures. Furthermore, it is possible to qualitatively extend the model by introducing stationary and transient states to describe the dynamic behavior of a MOS gas sensor using a temperature cyclic operation (TCO). This operation method can be used to increase the sensitivity of the sensor over several magnitudes [20]. It reduces the sensor drift considerably [21], allows a reliable quantitative calibration under ambient conditions [22].

Nevertheless, the simplified model is limited in its descriptive value. It neglects, or better hides, concepts of solid-state physics such as discrete energy levels or the band model in semiconductors. The model does not require a semiconducting material in order to measure reducing or oxidizing gases. Finally, the derivation of the model assumes that the amount of charge follows a continuous function and therefore neglects that there are no fractions of elementary charges. While these aspects are essential for a true scientific understanding of MOS gas sensors, they are not required for explaining the results of the experiments which the high school students perform.

\section{$5 \quad$ Experimental Implementation}

In order to experimentally support the concepts of the simplified model for the description of the functionality of a MOS gas sensor, a student laboratory experiment was developed. In this experiment the students investigate the reaction of a MOS gas sensor in the presence of different substances. The experiment is realized as an interactive HTML-based self-learning course divided into two practical and two theoretical sections. Within the practical parts measurements are performed in the headspace above the liquids water, apple juice and non-alcoholic beer. Based on the experimental observations, the simplified sensor model and its implications are developed step by step in the theoretical parts. The model is used both qualitatively and semiquantitatively. In order to avoid a cognitive overload of the students, formalquantitative treatment is avoided.

\section{$5.1 \quad$ Technical realization}

A simple circuit board was developed as basis for the learning setting, see Figs. 11 Fig. 12. Mounted on the bottom is the MOS gas sensor AS-MLV-P2 [23], which can be heated to defined temperatures via an integrated heater below the sensitive layer. The heater voltage is regulated by two potentiometers (coarse and fine) in the interval 0.7 - 3 Volt, corresponding to a sensor temperature range between $50^{\circ} \mathrm{C}$ and $400^{\circ} \mathrm{C}$. The voltage is smoothed by a capacitor $(10 \mu \mathrm{F})$. Heating voltage and the electrical resistance of the sensitive layer can be measured with multimeters connected to the board. The voltage is supplied by a standard micro USB connector, power is indicated by an LED. 

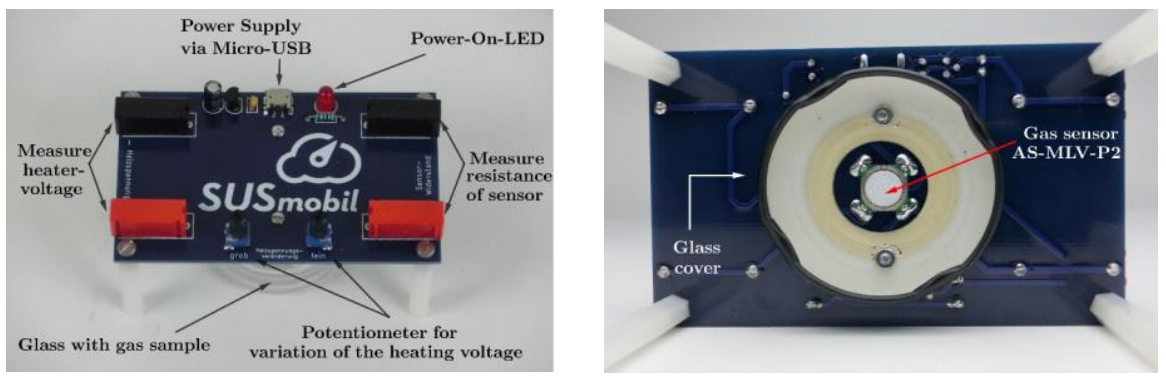

Fig. 11. Top and bottom view of the experimental circuit board. The gas sensor is mounted on the bottom, all other components on the top of the board.

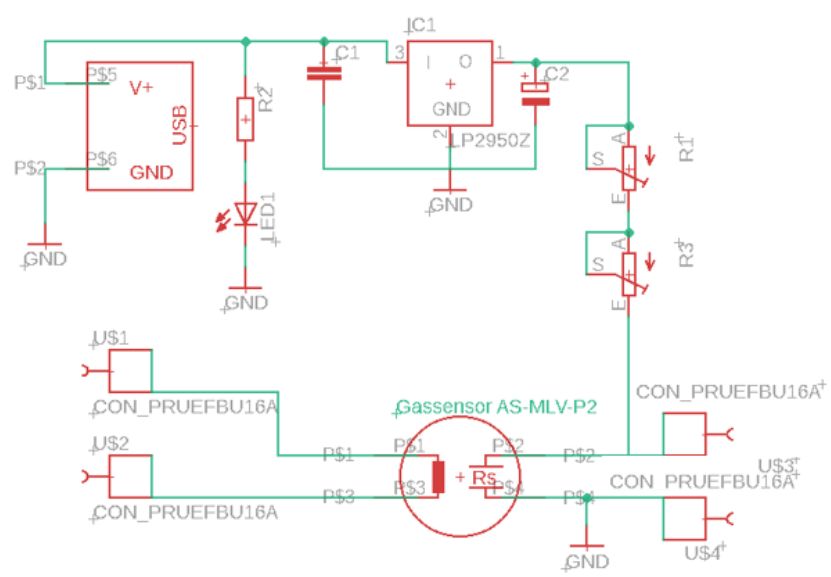

Fig. 12. Schematic diagram of the circuit board.

The samples are in liquid phase in small glass jars $(30 \mathrm{ml})$. The headspace (atmosphere above the liquid) through evaporation contains gaseous components in equilibrium with the liquid. The jars can be screwed underneath the circuit board, so that the sensor is in a sealed volume in the headspace of the liquid, see Fig. 13. The gas concentration in the headspace is determined by the concentration of the components in the liquid, for a pure liquid, e.g. water, the gas atmosphere is saturated. More importantly, the concentration of the individual gas components is constant at constant ambient temperature. 

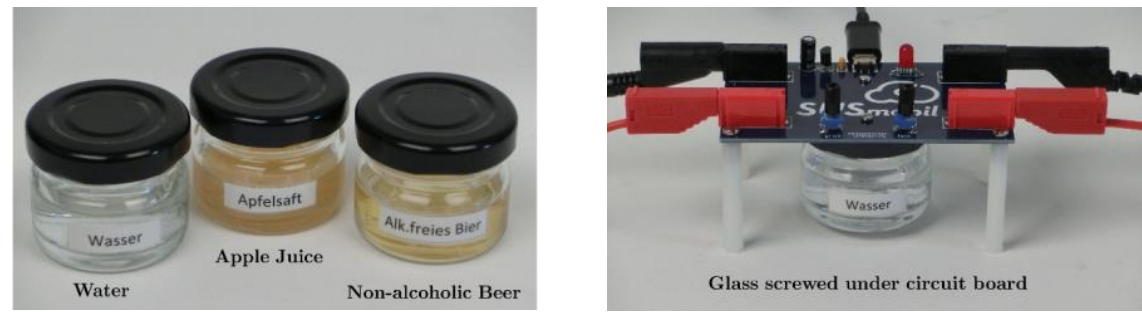

Fig. 13. Samples and attachment to the circuit board. The heating voltage for the gas sensor is provided by a USB power supply and can be manually varied by potentiometer and measured with a second multimeter.

\subsection{Experimental part I - Comparison of the sensor reaction at two sensor temperatures}

After a temperature change, a new equilibrium between adsorbed and desorbed oxygen is established on the surface. The duration of this process will be between milliseconds and hours [21]. In order to obtain comparable measurement results, the following measurements are therefore always performed at the same time intervals after a temperature change.

In the first experimental part, the students investigate the sensor reaction in the presence of water, apple juice and non-alcoholic beer at two different heating voltages $(1.2$ and $2.6 \mathrm{~V})$ corresponding to sensor temperatures of $125^{\circ} \mathrm{C}$ and $340^{\circ} \mathrm{C}$. The measurements are taken 60 seconds after a temperature change. Exemplary results are shown in Fig. 14.

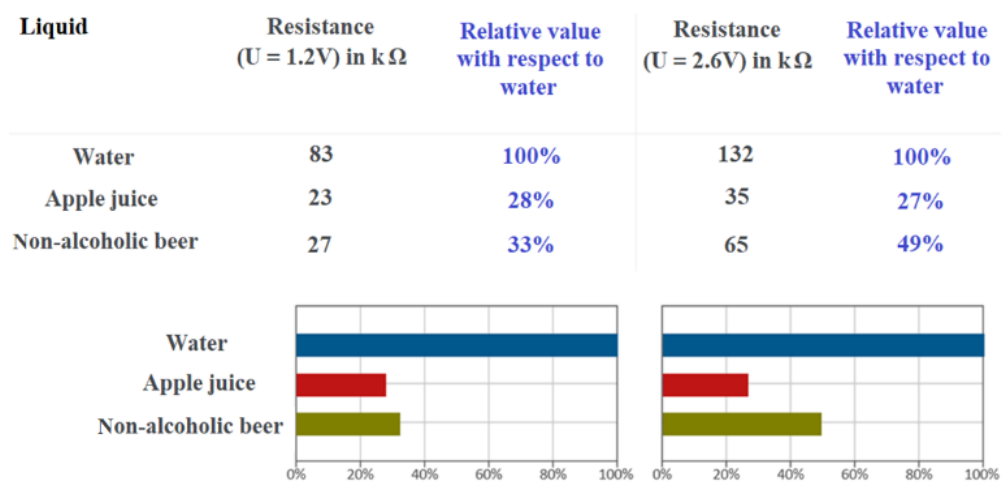

Fig. 14. Absolute sensor resistances (black) in the headspace for three test substances (water, apple juice, non-alcoholic beer) and values relative to water (blue) at heating voltages

of $1.2 \mathrm{~V}\left(125^{\circ} \mathrm{C}\right.$, left $)$ and $2.6 \mathrm{~V}\left(340^{\circ} \mathrm{C}\right.$, right $)$ recorded 60 seconds after a temperature change. A heating voltage of $2.6 \mathrm{~V}$ allows better discrimination of the three test substances. 
The measurements show the students that the sensor reaction is dependent on substance as well as temperature. At both sensor temperatures, it is shown that the sensor resistance above water is highest. The atmospheres above apple juice and nonalcoholic beer contain fairly large quantities of volatile organic compounds, primarily reducing gases like ethanol, which are in both cases responsible for the reduced resistances compared to water. At a heating voltage of $1.2 \mathrm{~V}$, the recorded signals, both absolute and relative to the sensor signal for water, are very similar, which does not allow reliable discrimination of apple juice and non-alcoholic beer. In contrast, all three test substances can be clearly distinguished at a heating voltage of $2.6 \mathrm{~V}$. This observation serves as motivation to investigate the temperature dependence of the sensor reaction more closely.

In order to understand why the sensor resistance is reduced in presence of a reducing gas, the simplified model is introduced in a first theoretical part of the course. This includes the theoretical description, as discussed in section III. at a constant sensor temperature.

\subsection{Experimental part II - Determination of a characteristic sensor reaction depending on its temperature}

In the second experimental part, the students investigate the temperature dependence of the sensor reaction in more detail. For this purpose, starting with a heating voltage of $2.8 \mathrm{~V}$, they reduce the sensor temperature in steps of $0.2 \mathrm{~V}$, and measure the sensor resistance in the presence of water and another substance of their choice. Measurements are taken 10 seconds after each a temperature change. The sensor signal for water represents the reference graph, as there are very few reducing substances in it. Exemplary measurement results are shown in Fig. 15.

The measurements show the expected trends derived from the physical grainboundary model as well as the simplified mechanistic model which both predict a minimum of the electrical resistance (a maximum of the conductance) at medium temperatures. In the case of the investigated MOS gas sensor AS-MLV-P2, this substance-dependent minimum is in the range between $150^{\circ} \mathrm{C}$ and $250^{\circ} \mathrm{C}$. The measurement curves are shifted to lower electrical resistance values (higher conductance values) in the presence of reducing gases, such as ethanol in apple juice, again as predicted by both models. 

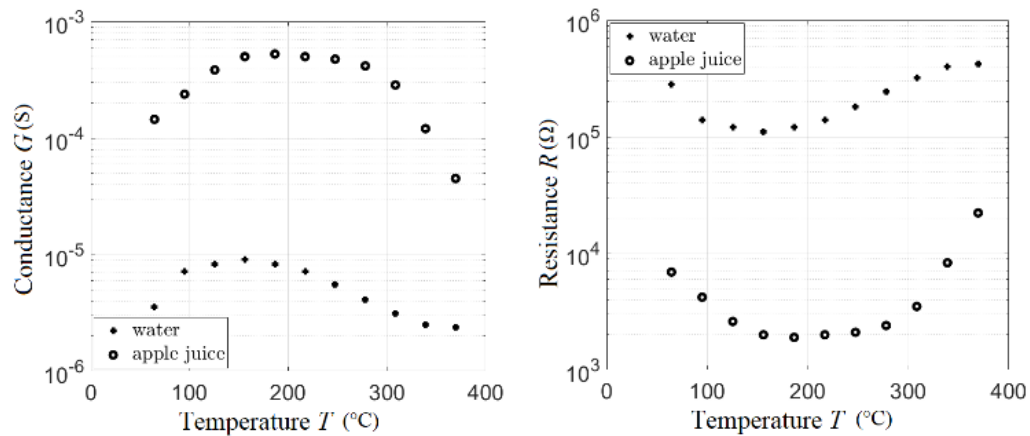

Fig. 15. Characteristic measurement curves of the sensor conductance (left) and resistance (right) as a function of the sensor temperature (determined by the heating voltage) in the headspace above water (dots) and apple juice (circles). Semi-logarithmic representation is used to better show the effects.

In the following second theoretical part the students extend the previously developed sensor model by the component of a changing temperature as shown in section III.

\subsection{Feedback and outlook}

Since 2018 the learning modules were conducted by various school classes of different age groups and have been continuously optimised based on feedback from teachers and students. By now it is a well-established part of the experiments offered by the student lab SinnTec at the Lab for Measurement Technology at Saarland University Saarbrücken and the Student Research Center Saarlouis. Most of the evaluations were very positive. Teachers commend the versatility of real and virtual parts of the experiment within a course. The structure as an HTML-based self-learning course on the PC with integrated data recording and presentation enables teachers and supervisors to deal exclusively with core problems of the students such as comprehension questions. This eliminates peripheral problems such as incorrect transcription of measurement results or problems with the graphical representation of measurement data. Furthermore, the experiments and concepts of gas sensor technology are a welcome change from the regular lessons and offer new insights for teachers, as gas sensor technology is not part of the teacher education programme in Germany.

By using the simplified sensor model, it is possible to reduce the complexity of the operation of a MOS gas sensor to the essential points. This makes it possible for students as young as grade 7 to gain an insight into the subject of gas sensors. This would otherwise only have been possible with a more advanced level of knowledge of physics and chemistry.

Students appreciate the versatile work on the PC. It has been shown that students compare measured values with each other, discuss possible deviations and look for reasons for this without being explicitly asked to do so. This can be interpreted as an indication of an increased interest in the topic of gas sensors. In a following self- 
learning course (module 2) the concepts developed in this module are taken up and continued in order to achieve a quantitative calibration of a sensor for one model substance (ethanol) at different concentrations. Here, the simplified model is extended to take dynamic effects into account which occur after a temperature step change. This leads to the introduction to the temperature cycled operation (TCO) of a MOS sensor which is used to increase the sensitivity and selectivity of the sensor [20]. Module 2 is described in detail in [24].

Both German and English versions of the HTML course can be downloaded as open source from the project website [25].

\section{Conclusion}

The developed simplified sensor model is adapted to the learning and knowledge level of high school students. It can describe the relevant processes taking place on the sensor surface as a function of the sensor temperature with and without reducing gas, both qualitatively and semi-quantitatively. The model describes the sensor reaction as the result of three overlaid and competing processes.

a. The velocity of the electrons increases with increasing temperature, based on the principle of an NTC thermistor, which leads to an increased conductance;

b. Oxygen molecules adsorb on the sensor surface capturing electrons, which means that fewer electrons are available for charge transport and, thus, a decrease of the conductance. Due to thermal activation of the adsorption the effect of the velocity of the electrons dominates at low temperatures, while oxygen adsorption dominates at high temperatures.

c. Reducing gas molecules can react with adsorbed oxygen freeing the captured electrons and, thus increasing conductance over the entire temperature range. Due to thermal activation, the effect of reducing gas is small at low temperature. At high temperature fast readsorption of oxygen in combination with the Weisz limit also results in a small change of the conductance for reducing gases.

This model is derived from experimental observations and developed theoretically step by step in a descriptive way using a self-learning course. Students study the sensor reaction to water, apple juice and non-alcoholic beer vapors as a function of the sensor temperature using a very simple set-up and record characteristic measurement graphs. This experiment shows that a combination of a few simple effects can lead to quite complex behavior and serves as an accessible introduction to the topic of MOS gas sensors.

\section{$7 \quad$ Acknowledgement}

The SUSmobil project was funded by the Deutsche Bundesstiftung Umwelt (DBU) within the framework of the funding line Education for Sustainable Development (German: Bildung für nachhaltige Entwicklung BNE). Special thanks go to Benjamin 
Brück, head of the Student Research Center Saarlouis, Germany, who helped develop the learning course. Further thanks to Josef Sniatecki, who provided significant support in optimizing the HTML code.

\section{References}

[1] European Environment Agency EEA, "EEA Report No 10/2019”, 2019.

Word Health Organization WHO," Burden of disease from ambient air pollution for 2012 - summary of results (2014)", [Online) Available:

http://www.who.int/phe/health_topics/outdoorair/databases/AAP_BoD_results_March2014. pdf.

[2] Jones, A.P. "Indoor air quality and health". Atmos. Environ, 33, pp. 4535-4564, 1999.

[3] Chang C. H. \& L. Pascua, "Singapore students' misconceptions of climate change." in International Research in Geographical and Environmental Education, 25(1), pp. 84-96, 2016. https://doi.org/10.1080/10382046.2015.1106206

[4] Rice, S. A., "Health effects of acute and prolonged CO2 exposure in normal and sensitive populations" in Proceedings Second Annual Conference on Carbon Sequestration, Alexandria, VA, May 5-8, 2003.

[5] Schütze, A. \& Sauerwald, T., "Indoor air quality monitoring”, in: E. Llobet (ed.): Advanced Nanomaterials for Inexpensive Gas Microsensors, pp. 209-234, 2020.https://doi.org/10.1016/b978-0-12-814827-3.00011-6

[6] Pettenkofer, M., "Besprechung allgemeiner auf die Ventilation bezüglicher Fragen, "Cotta, 1858.

[7] Schütze, A., Höfner, S., Brück, B., Hirth, M. \& Kuhn, J., "Citizen science with smart gas sensors: air quality assessment and environmental studies by students", in Proceedings IMCS 2018, 17th International Meeting on Chemical Sensors, Vienna, Austria, July 15-19, 2018. https://doi.org/10.5162/imcs2018/me.5

[8] Höfner, S., Schütze, A., Hirth, M., Kuhn, J. \& Brück, B., "Citizen Science für Schüler*innen: Durchführung von Umweltstudien mit Smartphone und mobiler Messtechnik', in Tagungsband, 20. GMA/ITG Fachtagung Sensoren und Messsysteme, pp. 476 481, 2019. https://doi.org/10.5162/sensoren2019/6.1.5

[9] Haklay, M., "Citizen Science and Volunteered Geographic Information - Overview and Typology of Participation in SUI", in Crowdsourcing Geographic Knowledge: Volunteered Geographic Information (VGI) in Theory and Practice, pp. 105-122, Berlin, 2013. https://doi.org/10.1007/978-94-007-4587-2_7

[10] Devictor, V., Whittaker, R \& Beltrame, C., "Beyond scarcity: citizen science as useful tools for conservation biogeography”, in Diversity and Distributions, Vol. 16 (3), pp. 354362, 2010. https://doi.org/10.1111/j.1472-4642.2009.00615.x

[11] Höfner, S. \& Schütze, A., "Umweltstudien mit Smartphone für Schüler*innen am Beispiel der Untersuchung der Luft in Bienenstöcken', in Tagungsband, 14. Dresdner Sensorsymposium, pp. 148-153, 2019 https://doi.org/10.5162/14dss2019/p1.12

[12] Madou, M. \& Morrison, S., "Chemical Sensing with Solid State Devices", in Academic Press, Boston, 1989.

[13] Ding, J., McAvoy, T., Cavicchi, R. \& Semancik, S.," Surface state trapping models for SnO2-based microhotplate sensors", in Sensors and Actuators B, Bd. 77, pp. 597-613, 2001. https://doi.org/10.1016/s0925-4005(01)00765-1

[14] Kappler, J.," Characterization of high performance $\mathrm{SnO} 2$ gas sensors for CO-detection by in situ techniques", Ph.D. dissertation Universität Tübingen, Germany, 2001. 
[15] Rantala T. \& Lantto, V., "Effects of mobile donors on potential distribution in grain contacts of sintered ceramic semiconductor', in Journal of Applied Physics, Bd. 79, p. 9206, 1996. https://doi.org/10.1063/1.362593

[16] Drude, P., "Zur Elektronentheorie der Metalle“, in Annalen der Physik, 306 (3), pp. 566613, 1900. https://doi.org/10.1002/andp.19003060312

[17] Feteira, A.," Negative Temperature Coefficient Resistance (NTCR) Ceramic Thermistors: An Industrial Perspective", in Journal of the American Ceramic Society, 92, pp. 967-983, 2009. https://doi.org/10.1111/j.1551-2916.2009.02990.x

[18] Geistlinger, H., "Electron theory of thin-film gas sensors", in Sensors and Actuators B: Chemical, 17 (1), pp. 47-60, 1993. https://doi.org/10.1016/0925-4005(93)85183-b

[19] Schultealbert, C., Baur, T., Schütze, S. \& Sauerwald, T, " Facile Quantification and Identification Techniques for Reducing Gases over a Wide Concentration Range Using a MOS Sensor in Temperature-Cycled Operation", in MDPI Sensors, 18:744, 2018. https://doi.org/10.3390/s18030744

[20] Gramm, A. \& Schütze, A.," High performance solvent vapor identification with a twosensor array using temperature cycling and pattern classification", in Sensors \& Actuators B, 95, pp. 58-65, 2003. https://doi.org/10.1016/s0925-4005(03)00404-0

[21] Schultealbert, C., Baur, T., Schütze, A., Böttcher, S. \& Sauerwald, T.,” A novel approach towards calibrated measurement of trace gases using metal oxide semiconductor sensors", in Sensors and Actuators B, 239, pp. 390-396, 2017. https://doi.org/10.1016/ j.snb.2016.08.002

[22] ams, „mouser” [Online]. Available: https://www.mouser.de/datasheet/2/588/AS-MLVP2_DS000359_1-00-1513213.pdf.

[23] Höfner, S., Hirth, M., Brück, B., Kuhn, J. \& Schütze, A.” Calibration of Metal Oxide Semiconductor Gas Sensors by High School Students", submitted in IEEE Transactions on Learning Technologies, 2020.

[24] [Online]. Available: http://www.susmobil.lmt.uni-saarland.de

[25] Arrhenius, S., "Über die Reaktionsgeschwindigkeit bei der Inversion von Rohrzucker durch Säuren, “in Z. Phys. Chem., 4, pp. 226-248, 1889. https://doi.org/10.1515/zpch$\underline{1889-0416}$

[26] Corless, R. M., Gonnet, G. H., Hare, D. E. G., Jeffrey, D. J. \& Knuth, D. E.” On the Lambert W Function", in Advances in Computational Mathematics, 5, pp. 329-359, 1996. https://doi.org/10.1007/bf02124750

\section{Authors}

Sebastian Höfner studied physics and received his diploma from the technical university Kaiserslautern in 2016. He is currently a research associate at the Lab for Measurement Technology (LMT) in the Department of Systems Engineering, Saarland University, Saarbrücken, Germany (e-mail: s.hoefner@1mt.uni-saarland.de). His research emphasis is the development of student experiments on air quality and the mentoring of environmental studies by high school students.

Dr. Michael Hirth studied physics, mathematics and astronomy at the Martin Luther University in Halle / Saale and received his Ph.D. in physics from TU Kaiserslautern in 2019. Since 2013 he is a research associate at the Physics Education Research Group in the Department of Physics, TU Kaiserslautern (e-mail: mhirth@physik.unikl.de). 
Benjamin Brück is head of the Student Research Center Saarlouis and teaches Physics and Mathematics at the Max-Planck-Gymnasium, Saarlouis, Germany.

Prof. Dr. Jochen Kuhn a full professor and head of the Physics Education Research Group at the Department of Physics, TU Kaiserslautern, Germany. He received his $\mathrm{PhD}$ in the field of physics in 2002 and his Habilitation in Physics Education in 2009, both at University of Koblenz-Landau. His research focuses on learning with multiple representations in STEM education in general and in physics education in particular using common and advanced multimedia technology (e-mail: kuhn@physik.uni-k1.de).

Prof. Dr. Andreas Schütze studied physics at the RWTH Aachen and received his Ph.D. in applied physics from JLU Gießen in 1994. Since 2000, he is full professor at Saarland University and head of the Laboratory for Measurement Technology. His research interests include microsensors and microsystems, especially advanced chemical sensor systems for environmental monitoring, security and control applications, as well as using sensors for outreach activities. (e-mail: schuetze@lmt.uni-saarland.de).

Article submitted 2020-10-14. Resubmitted 2020-12-23. Final acceptance 2020-12-25. Final version published as submitted by the authors. 


\section{Appendix}

The graphs in sections III and IV are based on the following derivations.

\subsection{Effect of sensor temperature - No atmosphere considered}

Theoretically, the dependence of the electrical conductance $G$ is interpreted by comparison to a thermistor with negative temperature coefficient (NTC). These temperature sensors are also based on semiconducting metal oxides and their electrical conductance $G$ increases with increasing temperature $T$ according to (14) [18] which also describes a MOS gas sensor [21].

$$
G \sim \exp \left(-\frac{E_{\mathrm{A}}^{\mathrm{Th}}}{k_{\mathrm{B}} T}\right)
$$

Here $E_{\mathrm{A}}^{\mathrm{Th}}$ is the activation energy to overcome the band gap, $k_{B}$ is the Boltzmann constant and $T$ is the temperature. Since no charge carriers are captured without an oxygen atmosphere, the total charge $Q$ remains constant, so that the increase of the electrical conductance $G$ according to (14) can be interpreted as an increase in the drift velocity of the electrons in (13).

\subsection{Influence of an oxygen atmosphere without reducing gas}

Due to the adsorption of oxygen on the sensor surface, free electrons $Q_{f}$ are captured. This reduces the number of charge carriers that are available for charge transport. As a result, the total charge $Q$ is reduced by the amount of bound charges $Q_{b}$.

$$
Q_{f}=Q-Q_{b}
$$

According to Arrhenius [26], the reaction of oxygen with the surface can be expressed by a temperature-dependent reaction rate $k$ :

$$
k=\exp \left(-\frac{E_{A}}{k_{B} T}\right)
$$

The term $E_{\mathrm{A}}$ describes the activation energy required for adsorption of oxygen on the surface. This allows the amount of bound charges to be calculated according to

$$
Q_{b}=Q \cdot \exp \left(-\frac{E_{A}}{k_{B} T}\right)
$$

As the surface coverage of negatively charged oxygen increases, so does the surface potential, and thus the required activation energy $E_{\mathrm{A}}$ also increases. This effect is known as "Weisz-Limit" [18]. In a first estimation, it is reasonable to assume a linear dependence for the surface coverage with a pre-factor $\alpha$.

$$
E_{A}\left(Q_{b}\right)=E_{A}^{0}+\alpha \cdot Q_{b}
$$


$E_{\mathrm{A}}^{0}$ describes the basic activation energy for adsorption of oxygen on the surface. After equilibration the surface coverage is determined as a function of temperature.

$$
Q_{b}=Q \cdot \exp \left(-\frac{E_{A}^{0}+\alpha \cdot Q_{b}}{k_{B} T}\right)
$$

The solution of this implicit equation with respect to $Q_{\mathrm{b}}$ is the analytical continuation of the Lambert $-\mathrm{W}$ - function [27]:

$$
Q_{\mathrm{b}}=\frac{k_{\mathrm{B}} T}{\alpha} \cdot W\left(\frac{\alpha \cdot Q \cdot \exp \left(-\frac{E_{\mathrm{A}}^{0}}{k_{\mathrm{B}} T}\right)}{k_{\mathrm{B}} T}\right)
$$

Using (15) the number of free charges is determined by

$$
Q_{f}=Q \cdot\left(1-\exp \left(-\frac{E_{A}^{0}+\alpha \cdot Q_{b}}{k_{B} T}\right)\right)
$$

and with (13) this results in a conductivity of the form

$$
G \sim Q \cdot\left(1-\exp \left(-\frac{E_{A}^{0}+\alpha \cdot Q_{b}}{k_{B} T}\right)\right) \exp \left(-\frac{E_{A}^{T h}}{k_{B} T}\right)
$$

\subsection{Influence of a reducing gas}

As a result of the reaction of reducing gases, fewer oxygen molecules are effectively adsorbed on the sensor surface. This phenomenon can be described by an effective activation energy $E_{A}^{e f f}$

$$
E_{A}^{0} \rightarrow E_{A}^{e f f}=E_{A}^{0}+E_{A}^{\prime}
$$

$E_{\mathrm{A}}^{\prime}$ is the activation energy for the reaction of a reducing gas with adsorbed oxygen on the surface, depending on both the substance and its concentration. Using (19) the number of bound charges can be derived by

$$
Q_{b}=Q \cdot \exp \left(-\frac{E_{A}^{0}+E_{A}^{\prime}+\alpha \cdot Q_{b}}{k_{B} T}\right)
$$

and therefore, the number of free charge carriers $Q_{\mathrm{f}}$ according to (15)

$$
Q_{f}=Q \cdot\left(1-\exp \left(-\frac{E_{A}^{0}+E_{A}^{\prime}+\alpha \cdot Q_{b}}{k_{B} T}\right)\right)
$$

With (13) this finally leads to the electrical conductance

$$
G \sim Q \cdot\left(1-\exp \left(-\frac{E_{A}^{0}+E_{A}^{\prime}+\alpha \cdot Q_{b}}{k_{B} T}\right)\right) \exp \left(-\frac{E_{A}^{T h}}{k_{B} T}\right)
$$

The electrical conductance decreases overall in the presence of reducing gases. For both low and high temperature, the conductance with and without reducing gas approach each other. This effect can also be observed in experiments (see section V). 